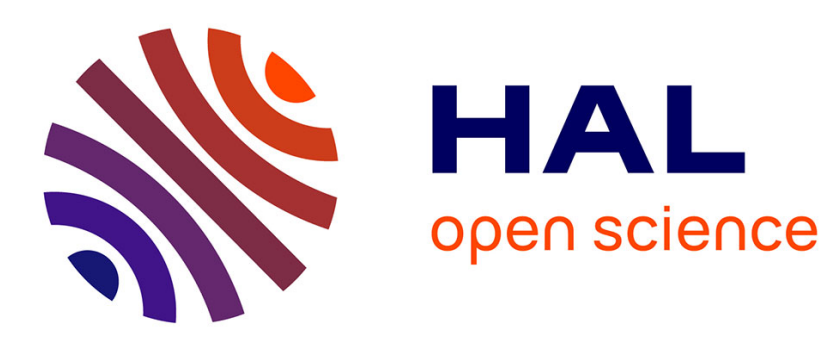

\title{
Explicit predictive control laws with a nonlinear constraints handling mechanism
}

Simona Dobre, Sorin Olaru, Didier Dumur

\section{To cite this version:}

Simona Dobre, Sorin Olaru, Didier Dumur. Explicit predictive control laws with a nonlinear constraints handling mechanism. International Conference on Computer as a tool, EUROCON 2007, Sep 2007, Warsaw, Poland. pp.CDROM. hal-00168609

\section{HAL Id: hal-00168609 \\ https://hal.science/hal-00168609}

Submitted on 29 Aug 2007

HAL is a multi-disciplinary open access archive for the deposit and dissemination of scientific research documents, whether they are published or not. The documents may come from teaching and research institutions in France or abroad, or from public or private research centers.
L'archive ouverte pluridisciplinaire HAL, est destinée au dépôt et à la diffusion de documents scientifiques de niveau recherche, publiés ou non, émanant des établissements d'enseignement et de recherche français ou étrangers, des laboratoires publics ou privés. 


\title{
Explicit predictive control laws with a nonlinear constraints handling mechanism
}

\author{
Simona Dobre*, Sorin Olaru ${ }^{\dagger}$, Didier Dumur ${ }^{\dagger}$ \\ * CRAN, Nancy - Université, CNRS UMR 7039,BP 239, F-54506 Vandœuvre-lès-Nancy Cedex, France, \\ Simona.Dobre@cran.uhp-nancy.fr \\ $\dagger$ Automatic Control Department - SUPELEC, 3 rue Joliot Curie, Gif-sur-Yvette, 91192, France, \\ \{sorin.olaru;didier.dumur\}@ supelec.fr
}

\begin{abstract}
This paper is dealing with the receding horizon optimal control techniques having as main goal the reduction of the computational effort inherent to the use of on-line optimization routines. The off-line construction of the explicit solution for the associated multiparametric optimization problems is advocated with a special interest in the presence of nonlinearities in the constraints description. The proposed approach is a geometrical one, based on the topology of the feasible domain. The resulting piecewise linear state feedback control law has to accept a certain degree of suboptimality, as it is the case for local linearizations or decompositions over families of parametric functions. In the presented techniques, this is directly related to the distribution of the extreme points on the frontier of the feasible domain.
\end{abstract}

Keywords-Predictive control, constraints, parameterized polyhedra.

\section{INTRODUCTION}

Conceiving optimal control policies implies the use of an infinite horizon for accounting the system behavior for coping with all the possible system evolutions. However, the infinite horizon will lead in a general case to an infinite number of optimization variables. Adding the presence of constraints and eventually the structural problems inherited from the model used for the prediction, one can have the picture of the difficulties in implementing such an infinite horizon optimal control algorithm.

The Model-based Predictive Control (MPC) is the practical methodology which uses an optimization based philosophy but only emulates the infinite horizon by sliding the prediction window [2], [3]. It leads by consequence to a tractable computational framework with interesting constraints handling capabilities.

The control action $u_{t}$ at state $x_{t}$ is obtained from the control sequence $\mathbf{k}_{u}^{*}=\left[u_{t}^{T}, \ldots, u_{t+N-1}^{T}\right]^{T}$ as a result of the optimization problem:

$$
\begin{array}{cl}
\min _{\mathbf{k}_{u}} & \varphi\left(x_{t+N}\right)+\sum_{k=0}^{N-1} l\left(x_{t+k}, u_{t+k}\right) \\
\text { subj. to : } & x_{t+1}=f\left(x_{t}\right)+g\left(x_{t}\right) u_{t} ; \\
& h\left(x_{t}, \mathbf{k}_{u}\right) \leq 0
\end{array}
$$

constructed for a finite prediction horizon $N$, cost per stage $l($.$) , terminal weight \varphi($.$) , the system dynamics$ described by $f(),. g($.$) and the constraints written in a$ compact form using elementwise inequalities on functions linking the states and the control actions, $h($.$) .$
The present paper deals with the case when $\varphi$ and $l$ are quadratic functions of $x$ and $\mathbf{k}_{u}, f$ and $g$ are linear, leading to an optimisation problem :

$$
\begin{gathered}
\mathbf{k}_{u}^{*}=\underset{\mathbf{k}_{u}}{\arg \min } 0.5 \mathbf{k}_{u}^{T} H \mathbf{k}_{u}+\mathbf{k}_{u}^{T} F x \\
\left\{\begin{array}{c}
A_{i n} \mathbf{k}_{u} \leq b_{i n}+B_{i n} x \\
f\left(x, \mathbf{k}_{u}\right) \leqslant 0
\end{array}\right.
\end{gathered}
$$

Unfortunately, the control sequence $\mathbf{k}_{u}^{*}$ is optimal only for a single initial condition - $x_{t}$ and produces an openloop trajectory which contrasts with the need for a feedback control law. This drawback is overcome by solving the local optimization (1) for every (measured) state, thus indirectly producing a state feedback law. However, taking into account that the measurements can be available faster than the optimal control sequence becomes available (as output of the optimization solver), an important information can be lost with irreversible consequences on the closed-loop performances.

The system state can be interpreted as a vector of parameters, and the problems to be solved are part of the multiparametric optimization programming family [4]. From the cost function point of view, the parametrization is somehow easier to deal with, the unconstrained optimum being $\mathbf{k}_{u}^{u c}=H^{-1} F x$. The parametrization of the feasible domain is more difficult to deal with. The approach adopted in the following uses the concept of parameterized polyhedra for the convex sets and Voronoi partitions of the parameters space for the non-convex case.

This is equivalent to constraints linearization, their activation with respect to the optimum search being then readily expressed [1] (as long as the Abadie's constraints qualification holds). Indeed, the KKT optimality conditions can be used for characterizing the partitions of the state space for the control law. From the computational point of view, this is the technique which assures the less computational effort [5]. From the point of view of the feasible domain topology, the dual representation generators/constraints can bring useful information.

One can find in the literature alternative techniques for explicit solution construction in the case of nonlinear systems. The main assumption consists in the convexity of the optimisation problem. In [6] a linearization is done over a specific class of functions (polynomial), in [7] a sampling of the parameters space is performed or one 
can replace directly the nonlinear bounds of the feasible domain by an approximated linearized one [8].

\section{FROM FINITE TIME OPTIMAL CONTROL TO MULTIPARAMETRIC OPTIMIZATION}

MPC implies the minimization of a cost index based on the predicted plant evolution. The first part of the optimal control sequence is effectively applied after the "receding horizon principle".

\section{A. Optimal control}

Consider the discrete linear time-invariant system in a state-space description:

$$
\left\{\begin{array}{l}
x_{t+1}=A x_{t}+B u_{t} \\
y_{t}=C x_{t}
\end{array}, \quad t \in \mathbb{N}^{+}\right.
$$

where $x_{t} \in \mathbb{R}^{n}$ and $u_{t} \in \mathbb{R}^{m}$ are the state and control vector. It is assumed throughout that the pair $(A, B)$ is stabilizable. At each sampling time, the current state (assumed to be available) $x_{t}=x_{t \mid t}$ can be used to find the optimal control sequence $\mathbf{k}_{u}^{*}=\left[u_{t \mid t}^{T}, \cdots, u_{t+N_{y}-1 \mid t}^{T}\right]^{T}$ :

$$
\begin{aligned}
\mathbf{k}_{u}^{*}= & \underset{\mathbf{k}_{u}}{\arg \min } x_{t+N_{y} \mid t}^{T} P x_{t+N_{y} \mid t}+ \\
& +\sum_{k=0}^{N_{y}-1}\left\{x_{t+k \mid t}^{T} Q x_{t+k \mid t}+u_{t+k \mid t}^{T} R u_{t+k \mid t}\right\}
\end{aligned}
$$

subject to:

$$
\left\{\begin{array}{l}
x_{t+k+1 \mid t}=A x_{t+k \mid t}+B u_{t+k \mid t}, k \geqslant 0 \\
g\left(u_{t+k \mid t}, x_{t+k \mid t}\right) \leqslant \gamma, 0 \leqslant k \leqslant N_{y}-1 \\
u_{t+k \mid t}=K_{L Q R} x_{t+k \mid t}+e, N_{u} \leqslant k \leqslant N_{y}-1
\end{array}\right.
$$

where $Q=Q^{T} \geqslant 0$ and $R=R^{T}>0$ are the weighting matrices and the pair $\left(Q^{1 / 2}, A\right)$ is detectable. The inequality constraints are fully described by the Lipschitz function, $g$ and by $\gamma \in \mathbb{R}^{q}$. The prediction horizon - $N_{y}$, the control horizon $N_{u} \leqslant N_{y^{-}}$together with $Q$ and $R$ are the knobs of the control design. $K_{L Q R}$ is the stabilizing feedback control gain for unconstrained linear systems calculated using the solution of a discrete algebraic Riccati equation:

$$
\begin{aligned}
& P=Q+A^{T} P A-K_{L Q R}^{T}\left(R+B^{T} P B\right) K_{L Q R} \\
& K_{L Q R}=\left(R+B^{T} P B\right)^{-1} B^{T} P A
\end{aligned}
$$

and $e$ is the new input vector, calculated as:

$$
e=\left(C\left(I-A+B K_{L Q R}\right)^{-1} B\right)^{-1} y_{c}
$$

with $y_{c}$ the desired output.

The optimization problem presented here is a modified version of the constrained infinite horizon linear quadratic problem where both the cost index and the inequality constraints are defined along an infinite prediction horizon. The fact that the explicit constraints cover here only the instants until $N_{y}$ is due to the implicit terminal conditions implying that the choice of $N_{y}$ is made such that the usage of $u_{t+k \mid t}=K_{L Q R} x_{t+k \mid t}+\mathrm{e}$ will not trespass the feasible region for $k \geqslant N_{y}$. Constructive methods exist to determine the appropriate $N_{y}$ for each specific system and set of constraints. The overall optimisation problem is tractable with standard quadratic methods as it has $p=m \cdot N_{y}$ decision variables and $(q+m) N_{y}$ constraints (with $q$ - the number of inequality constraints).

Compacting the state involved in the optimization problem (2) as $\mathbf{x}=\left[x_{t+1 \mid t}^{T}, \cdots, x_{t+N_{y} \mid t}^{T}\right]^{T}$ and noting $x=x_{t \mid t}$ the cost function could be rewritten in the matrix formulation as:

$$
\mathbf{k}_{u}^{*}=\arg \min _{\mathbf{k}_{u}} x^{T} Q x+\mathbf{x}^{T} \bar{Q} \mathbf{x}+\mathbf{k}_{u}^{T} \bar{R} \mathbf{k}_{u}
$$

subject to :

$$
\left\{\begin{array}{c}
h\left(x, \mathbf{x}, \mathbf{k}_{u}\right) \leqslant \bar{\gamma} \\
J \cdot \mathbf{k}_{u}=\bar{K} \cdot \mathbf{x}+\bar{e}
\end{array}\right.
$$

where $\bar{Q}=\operatorname{diag}[Q, \cdots, Q, P] \in \mathbb{R}^{N_{y} n \times N_{y} n}, \bar{R}=$ $\operatorname{diag}[R, \cdots, R] \in \mathbb{R}^{N_{y} m \times N_{y} m}$,

$$
\begin{aligned}
& \bar{K}=\left[\mathbf{0}_{\left(N_{y}-N_{u}\right) m \times N_{u} n} \quad \operatorname{diag}\left[K_{L Q R}, \cdots, K_{L Q R}\right]\right] \\
& J=\left[\begin{array}{ll}
\mathbf{0}_{\left(N_{y}-N_{u}\right) m \times N_{u} m} & \mathbf{I}_{\left(N_{y}-N_{u}\right) m}
\end{array}\right] \\
& \bar{e}=\left[\begin{array}{c}
e \\
\vdots \\
e
\end{array}\right] \in \Re^{\left(N_{y}-N_{u}\right) m \times 1}, \bar{\gamma}=\left[\begin{array}{c}
\gamma \\
\gamma \\
\vdots \\
\gamma
\end{array}\right] \in \Re^{N_{y} q \times 1} \\
& h\left(x, \mathbf{x}, \mathbf{k}_{u}\right)=\left[\begin{array}{c}
g\left(x, u_{t \mid t}\right) \\
\vdots \\
g\left(x_{t+N_{y}-1 \mid t}, u_{t+N_{y}-1 \mid t}\right)
\end{array}\right] .
\end{aligned}
$$

The system's state evolution can be transformed in a compact form:

$$
\mathbf{x}=\Phi x+\Gamma \mathbf{k}_{u}
$$

with

$$
\Phi=\left[\begin{array}{c}
A \\
A^{2} \\
\vdots \\
A^{N_{y}}
\end{array}\right] \in \Re^{N_{y} n \times n}
$$

and

$$
\Gamma=\left[\begin{array}{ccccc}
B & \mathbf{0}_{n \times m} & \mathbf{0}_{n \times m} & \cdots & \mathbf{0}_{n \times m} \\
A B & B & \mathbf{0}_{n \times m} & \cdots & \mathbf{0}_{n \times m} \\
A^{2} B & A B & B & \cdots & \mathbf{0}_{n \times m} \\
\vdots & \vdots & \vdots & \ddots & \vdots \\
A^{N_{y}-1} B & A^{N_{y}-2} B & A^{N_{y}-3} B & \cdots & B
\end{array}\right]
$$

The optimization problem (2) within MPC becomes:

$$
\mathbf{k}_{u}^{*}=\underset{\mathbf{k}_{u}}{\arg \min } \mathbf{k}_{u}^{T} H \mathbf{k}_{u}+2 \mathbf{k}_{u}^{T} F x+x^{T} G x
$$

subject to

$$
\left\{\begin{array}{c}
f\left(x, \mathbf{k}_{u}\right) \leqslant b_{i n} \\
A_{e q} \mathbf{k}_{u}=b_{e q}+B_{e q} x
\end{array}\right.
$$

with $H=\bar{R}+\Gamma^{T} \cdot \bar{Q} \cdot \Gamma, F=\Gamma^{T} \cdot \bar{Q} \cdot \Phi, G=Q+\Phi^{T} \cdot \bar{Q} \cdot \Phi$, $b_{i n}=\bar{\gamma}, A_{e q}=J-\bar{K} \cdot \Gamma, B_{e q}=\bar{K} \cdot \Phi, b_{e q}=\bar{e}$ and $f\left(x, \mathbf{k}_{u}\right)=h\left(x, \Phi \cdot x+\Gamma \cdot \mathbf{k}_{u}, \mathbf{k}_{u}\right)$. 


\section{PARAMETRIZATION OF POLYHEDRAL DOMAINS}

\section{A. Double representation}

A mixed system of linear equalities and inequalities defines a polyhedron [9]. In the parameter free case, it is represented by the equivalent dual (Minkowski) formulation:

$$
\begin{aligned}
& \mathcal{P}=\left\{\mathbf{k}_{u} \in \mathbb{R}^{p} \mid A_{e q} \mathbf{k}_{u}=b_{\text {eq }} ; A_{\text {in }} \mathbf{k}_{u} \leq b_{\text {in }}\right\} \\
& \Longleftrightarrow \mathcal{P}=\underbrace{\text { conv.hull } \mathbf{V}+\text { cone } \mathbf{R}+\text { lin.space } \mathbf{L}}_{\text {generators }}
\end{aligned}
$$

where conv.hull $\mathbf{V}$ denotes the set of convex combinations of vertices $\mathbf{V}=\left\{\mathbf{v}_{1}, \ldots, \mathbf{v}_{\vartheta}\right\}$, cone $\mathbf{R}$ denotes nonnegative combinations of unidirectional rays in $\mathbf{R}=$ $\left\{\mathbf{r}_{1}, \ldots, \mathbf{r}_{\rho}\right\}$ and lin.space $\mathbf{L}=\left\{\mathbf{l}_{1}, \ldots, \mathbf{l}_{\lambda}\right\}$ represents a linear combination of bidirectional rays (with $\vartheta, \rho$ and $\lambda$ the cardinals of the related sets). This dual representation [12] in terms of generators can be rewritten as:

$$
\begin{gathered}
\mathcal{P}=\left\{\mathbf{k}_{u} \in \mathbb{R}^{p} \mid \mathbf{k}_{u}=\sum_{i=1}^{\vartheta} \alpha_{i} \mathbf{v}_{i}+\sum_{i=1}^{\rho} \beta_{i} \mathbf{r}_{i}+\sum_{i=1}^{\lambda} \gamma_{i} \mathbf{l}_{i} ;\right. \\
\left.0 \leq \alpha_{i} \leq 1, \sum_{i=1}^{\vartheta} \alpha_{i}=1, \beta_{i} \geq 0, \forall \gamma_{i}\right\}
\end{gathered}
$$

with $\alpha_{i}, \beta_{i}, \gamma_{i}$ the coefficients describing the convex, nonnegative and linear combinations in (10).

Numerical methods like the Chernikova algorithm [10] are implemented for constructing the double description, either starting from constraints (10) either from the generators (11) representation.

\section{B. The parametrization}

A parameterized polyhedron [11] is defined in the implicit form by a finite number of inequalities and equalities with the note that, in both cases, the affine part depends linearly on a vector of parameters $x \in \mathbb{R}^{n}$ :

$$
\begin{array}{r}
\mathcal{P}(x)=\left\{\mathbf{k}_{u}(x) \in \mathbb{R}^{p} \mid A_{e q} \mathbf{k}_{u}=B_{e q} x+b_{e q} ;\right. \\
\left.A_{i n} \mathbf{k}_{u} \leq B_{i n} x+b_{i n}\right\}
\end{array} \quad \begin{array}{r}
\vartheta \\
=\left\{\mathbf{k}_{u}(x) \mid \mathbf{k}_{u}(x)=\sum_{i=1}^{\vartheta} \alpha_{i}(x) \mathbf{v}_{i}(x)\right. \\
\left.+\sum_{i=1}^{\rho} \beta_{i} \mathbf{r}_{i}+\sum_{i=1}^{\lambda} \gamma_{i} \mathbf{l}_{i}\right\} \\
0 \leq \alpha_{i}(x) \leq 1, \sum_{i=1}^{\vartheta} \alpha_{i}(x)=1, \beta_{i} \geq 0, \forall \gamma_{i} .
\end{array}
$$

This dual representation of the parameterized polyhedral domain reveals the fact that only the vertices are concerned by the parametrization (resulting the so-called parameterized vertices $-\mathbf{v}_{i}(x)$ ), whereas the rays and the lines do not change with the parameter's variation. In order to effectively use the generators representation in (12), several aspects have to be clarified regarding the parametrization of the vertices (see for exemple [11] and the geometrical toolboxes like POLYLIB [13]).

Indeed the projections are to be computed for all the $n$ faces, those which are degenerated are to be discarded and all the others are stored as validity domains $-D_{\mathbf{v}_{i}} \in \mathbb{R}^{n}$, for the parameterized vertices that they are identifying:

$$
D_{\mathbf{v}_{i}}=\operatorname{Proj}_{n}\left(F_{j}^{n}(\tilde{P})\right)
$$

Once the parameterized vertices are identified and their validity domain stored, the dependence on the parameters vector can be found using the supporting hyperplanes for each $n$-face:

$$
\mathbf{v}_{i}(x)=\left[\begin{array}{c}
A_{e q} \\
\bar{A}_{i n_{j}}
\end{array}\right]^{-1}\left[\begin{array}{c}
B_{e q} \\
\bar{B}_{i n_{j}}
\end{array}\right] x+\left[\begin{array}{c}
b_{e q} \\
\bar{b}_{i n_{j}}
\end{array}\right]
$$

where $\bar{A}_{i n_{j}}, \bar{B}_{i n_{j}}, \bar{b}_{i n_{j}}$ represent the subset of the inequalities, satisfied by saturation for $F_{j}^{n}(\tilde{P})$. The inversion is well defined as long as the faces with degenerate projections are discarded.

\section{MultiparametriC OPTIMIZATION}

In (9), the optimization problem acts in the space of future control actions while the current state vector $x$ influences the unconstrained optimum and the affine part of the constraints, acting as a parameter. This dependance transforms the classical optimization problem in a multiparametric one (the use of "multiparametric" syntagm is for discriminating the case when the parameter is scalar).

\section{A. A mature framework}

A popular class of control problems described by (9) presents quadratic cost functions and linear constraints:

$$
\begin{aligned}
& \mathbf{k}_{u}^{*}=\arg \min _{\mathbf{k}_{u}} 0.5 \mathbf{k}_{u}^{T} H \mathbf{k}_{u}+\mathbf{k}_{u}^{T} F x \\
& \text { subject to: } \quad A_{i n} \mathbf{k}_{u} \leq b_{i n}+B_{i n} x
\end{aligned}
$$

This is known in the literature as the multiparametric quadratic problem (mpQP) and its optimality conditions (Karush-Kuhn-Tucker) are resumed by:

- Primal feasibility:

$$
A_{i n} \mathbf{k}_{u}^{*} \leqslant B_{\text {in }} x+b_{\text {in }}
$$

- Dual feasibility:

$$
\left\{\begin{array}{l}
H \mathbf{k}_{u}^{*}+F^{T} x+A_{i n}^{T} \mu=0 \\
\mu \geqslant 0
\end{array}\right.
$$

- Complementary slackness:

$$
\mu^{T}\left(A_{i n} \mathbf{k}_{u}^{*}-B_{i n} x-b_{i n}\right)=0
$$

\section{B. Explicit solution for $m p Q P$}

Exploiting these conditions the global solution can be explicitly represented by a piecewise linear and continuous function [14], [15]:

$$
\mathbf{k}_{u}^{*}(x)=K_{i} * x+\kappa_{i}, \text { for } x \in D_{i}, i \in \mathcal{I}
$$

with $\mathcal{I} \subset \mathbb{N}$ the index set for all combinations of constraints $\left(\bar{A}_{i n}, \bar{B}_{i n}, \bar{b}_{i n}\right)$ saturated by a local optimum. The linear dependence is assured by:

$$
\begin{aligned}
K_{i}= & H^{-1} \bar{A}_{i n}^{T}\left(\bar{A}_{i n} H^{-1} \bar{A}_{i n}^{T}\right)^{-1}\left(\bar{B}_{i n}+\bar{A}_{i n} H^{-1} F^{T}\right)- \\
& \quad-H^{-1} F^{T} ; \\
\kappa_{i}= & H^{-1} \bar{A}_{i n}^{T}\left(\bar{A}_{i n} H^{-1} \bar{A}_{i n}^{T}\right)^{-1} b_{i n},
\end{aligned}
$$

and the regions $D_{i}$ are convex polyhedra in $\mathbb{R}^{n}$ defined by:

$$
\left\{\begin{array}{l}
\left(A_{i n} K_{i}-B_{i n}\right) x \leq b_{i n}-\kappa_{i} \\
-\left(\bar{A}_{i n} H^{-1} \bar{A}_{i n}^{T}\right)^{-1}\left(\bar{A}_{i n} H^{-1} F^{T} x+\bar{b}_{i n}+\bar{B}_{i n} x\right) \geq 0
\end{array}\right.
$$


the second set of inequalities being originated by the positiveness of the Lagrange multipliers $\left(\mu_{i} \geq 0\right)$.

MPC uses only the first component of this optimal solution:

$$
u^{M P C}(x)=K_{i}^{M P C} * x+\kappa_{i}^{M P C} \text {, with } i \text { s.t. } x \in D_{i},
$$

and $K_{i}^{M P C}, \kappa_{i}^{M P C}$ the first components of $K_{i}, \kappa_{i}$.

\section{A topological approach}

The piecewise linear structure of the optimal solution can be obtained also by using the parameterized vertices (14) associated to the set of active constraints [16]. In this case the main idea is to consider the unconstrained optimum:

$$
\mathbf{k}_{u}^{u c}\left(x_{t}\right)=H^{-1} F x_{t}
$$

and its position with respect to the feasible domain given by a parameterized polyhedron as in (12).

If a simple transformation is performed:

$$
\tilde{\mathbf{k}}_{u}=H^{1 / 2} \mathbf{k}_{u}
$$

then the isocost curves of the quadratic function are transformed from ellipsoid into circles centered in $\tilde{\mathbf{k}}_{u}^{u c}\left(x_{t}\right)=$ $H^{-1 / 2} F x_{t}$. Further one can use the Euclidean projection in order to retrieve the explicit solution. Indeed if the unconstrained optimum $\tilde{\mathbf{k}}_{u}^{u c}\left(x_{t}\right)$ is contained in the feasible domain $\tilde{\mathcal{P}}\left(x_{t}\right)$ then it is also the solution of the constrained case, otherwise existence and uniqueness are assured as follows:

Proposition: For any exterior point $\tilde{\mathbf{k}}_{u}\left(x_{t}\right) \notin \tilde{\mathcal{P}}\left(x_{t}\right)$, there exists an unique point characterized by a minimal distance with respect to $\tilde{\mathbf{k}}_{u}^{u c}\left(x_{t}\right)$. This point satisfies:

$$
\left(\tilde{\mathbf{k}}_{u}^{u c}\left(x_{t}\right)-\tilde{\mathbf{k}}_{u}^{*}\left(x_{t}\right)\right)^{T}\left(\tilde{\mathbf{k}}_{u}-\tilde{\mathbf{k}}_{u}^{*}\left(x_{t}\right)\right) \leqslant 0, \forall \tilde{\mathbf{k}}_{u} \in \tilde{\mathcal{P}}\left(x_{t}\right) \square
$$

The construction mechanism uses the parameterized vertices in order to split the regions neighboring the feasible domain in zones characterized by the same type of projection. In figure 1 a simple example is given starting from the parameterized polyhedron $\mathcal{P}$ in the extended space (argument and parameters), the regions with the same type of projection are found and finally the explicit solution in terms of a piecewise linear function of parameters is obtained.

\section{HANDLING NONLINEARITIES}

Consider now the case of mixed type of constraints (linear/nonlinear):

$$
\begin{gathered}
\mathbf{k}_{u}^{*}=\underset{\mathbf{k}_{u}}{\arg \min } 0.5 \mathbf{k}_{u}^{T} H \mathbf{k}_{u}+\mathbf{k}_{u}^{T} F x \\
\left\{\begin{array}{c}
h\left(x, \mathbf{k}_{u}\right) \leqslant 0 \\
A_{\text {in }} \mathbf{k}_{u} \leq b_{\text {in }}+B_{\text {in }} x
\end{array}\right.
\end{gathered}
$$

An important point to note is the quadratic form of the cost function which offers interesting structural properties (convexity, uniqueness of the unconstrained optimum). This is not restricting the generality for control systems described by (3) as long as the usual choice for the cost function is the "sum of squared error weighted by (norm 2) control effort".

\section{A. Optimality conditions for nonlinear constraints}

Let $\bar{x}$ be a feasible parameter vector. The KKT optimality conditions can still be formulated as:

- Primal feasibility:

$$
\left\{\begin{array}{l}
h\left(\bar{x}, \mathbf{k}_{u}\right) \leqslant 0 \\
A_{i n} \mathbf{k}_{u} \leq b_{i n}+B_{i n} \bar{x}
\end{array}\right.
$$

- Dual feasibility:

$$
\left\{\begin{array}{l}
H \mathbf{k}_{u}+F^{T} \bar{x}+A_{i n}^{T} \mu+\nabla_{\mathbf{k}_{u}} h\left(\bar{x}, \mathbf{k}_{u}\right)^{T} \nu=0 \\
\mu \geqslant 0, \nu \geqslant 0
\end{array}\right.
$$

- Complementary slackness:

$$
\left[\mu^{T} \nu^{T}\right]\left[\begin{array}{c}
A_{i n} \mathbf{k}_{u}-B_{i n} \bar{x}-b_{i n} \\
h\left(\bar{x}, \mathbf{k}_{u}\right)
\end{array}\right]=0
$$

The difference resides in the fact that the KKT conditions are only necessary and not sufficient for optimality due to the presence of nonlinearity.

\section{B. The topology of the feasible domain}

Indeed the sufficiency is lost due to the lack of constraint qualification (the Abadie constraint qualification holds automatically for the linear constraints but needs additional assumptions for the nonlinear case, see the next theorem).

Theorem (KKT sufficient conditions) [1]: Let $x=\bar{x}$ and the associated feasible domain $\mathbf{U}(\overline{\mathbf{x}})$ be a nonempty set in $\mathbb{R}^{N_{y} m}$ described by the constraints in (23), with $h_{i}\left(\bar{x}, \mathbf{k}_{u}\right): \mathbb{R}^{N_{y} m} \rightarrow \mathbb{R}$, the components of $h\left(\bar{x}, \mathbf{k}_{u}\right)$. Let $\mathbf{k}_{u}^{*} \in \mathbf{U}(\overline{\mathbf{x}})$ and let $\mathcal{I}=\left\{i: h_{i}\left(\bar{x}, \mathbf{k}_{u}^{*}=0\right)\right\}, \mathcal{J}=$ $\left.\left\{j: A_{i n_{j}} \mathbf{k}_{u}^{*}-B_{i n_{j}} \bar{x}-b_{i n_{j}}=0\right)\right\}$. Suppose the KKT conditions hold, such that:

$$
H \mathbf{k}_{u}^{*}+F^{T} \bar{x}+\sum \mu_{j} A_{i n_{j}}^{T}+\sum \nu_{i} \nabla_{\mathbf{k}_{u}} h_{i}\left(\bar{x}, \mathbf{k}_{u}^{*}\right)^{T}=0
$$

If $h_{i}$ is quasiconvex at $\mathbf{k}_{u}^{*}$ for $i \in \mathcal{I}$, then this is a global solution to the problem (23) $\square$

Due to these problems, up to date, the explicit solutions for the general nonlinear multiparametric programming case were not tackled. Only for convex nonlinearities approximate explicit solutions were proposed [7].

In the following a solution based on linear approximation of feasible domains is proposed. This will answer the question regarding the optimality of a solution with piecewise linear structure.

\section{Preliminaries for linear approximations of mixed lin- ear/nonlinear feasible domains}

The idea is to exploit the existence of linear constraints in (23) and construct exact solutions as long as the unconstraint optimum can be projected on them. In a second stage if the unconstrained optimum is projected on the convex part of the nonlinear constraints, then an approximate solution is obtained by their linearization. Finally if the unconstrained optimum has to be projected on the nonconvex nonlinear constraints then a Voronoi partition is used to construct the explicit solution.

Before detailing the algorithms several useful tools have to be introduced: 


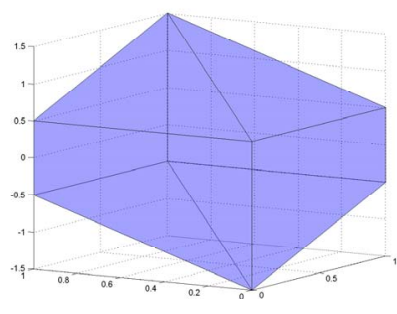

(a) A feasible domain as a parameterized polyhedron

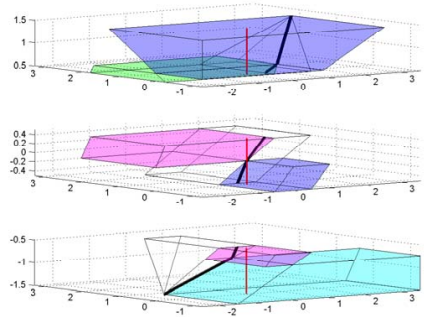

(b) The neighboring zones with different projection laws

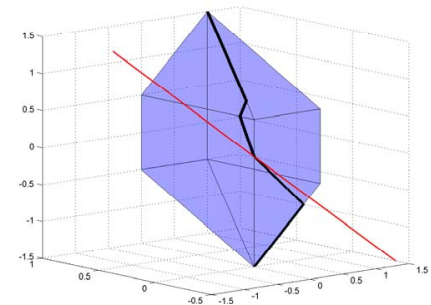

(c) Explicit solution as a piecewise linear function

Fig. 1. From the feasible domain as a parameterized polyhedron to the explicit solution

1) Gridding of the parameter space: The parameters (state) space is sampled in order to obtain a representative grid $\mathcal{G}$. The way of distributing the points in the state space may follow a uniform distribution, logarithmic or tailored according to the a-priori knowledge of the nonlinearities.

Figure 2 illustrates two common types of gridding, the linear and the spherical one (note that the linear distribution of points can be very conservative for large dimensions spaces).

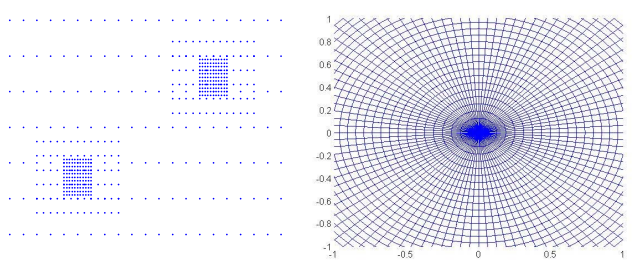

Fig. 2. Example of grid of the parameters space

For each point of the grid $x \in \mathcal{G}$ a set of points on the frontier of the feasible domain $D(x)$ can be obtained $\mathcal{V}_{x}$ by the same kind of parceling. By collecting $\mathcal{V}_{x}$ for all $x \in \mathcal{G}$ a distribution of points $\mathcal{V}_{\mathcal{G}}$ in the extended arguments+parameters space is obtained.

2) Convex hulls: A basic operation is the construction of the convex hull (or a convenable approximation) for the feasible domain in (23). Writing this parameterized feasible domain as:

$$
D(x)=\left\{\begin{array}{l|c}
\mathbf{k}_{u} \mid \begin{array}{c}
h\left(x, \mathbf{k}_{u}\right) \leqslant 0 \\
A_{i n} \mathbf{k}_{u} \leq b_{\text {in }}+B_{\text {in }} x
\end{array}
\end{array}\right\}
$$

and using the distribution of points on the frontier $\mathcal{V}_{\mathcal{G}}$, one can define in the extended (argment+parameters) space a convex hull $\mathcal{C}_{\mathcal{V}_{\mathcal{G}}}$ :

$$
\begin{aligned}
\mathcal{C}_{\mathcal{V}_{\mathcal{G}}}=\left\{\left[\begin{array}{c}
\mathbf{k}_{u} \\
x
\end{array}\right] \in \mathbb{R}^{m N_{y}+n} \mid \exists\left[\begin{array}{c}
\mathbf{k}_{u_{i}} \\
x_{i}
\end{array}\right],\right. \\
i=1 . . m N_{y}+n+1, \mathbf{k}_{u_{i}} \in \mathcal{V}_{\mathcal{G}}, \\
\text { t.q. } \quad\left[\begin{array}{c}
\mathbf{k}_{u} \\
x
\end{array}\right]=\sum_{i=1}^{m N_{y}+n+1} \lambda_{i}\left[\begin{array}{c}
\mathbf{k}_{u_{i}} \\
x_{i}
\end{array}\right], \\
\left.\sum_{i=1}^{m N_{y}+n+1} \lambda_{i}=1 ; \lambda_{i} \geq 0\right\}
\end{aligned}
$$

3) Voronoi partition: The Voronoi partition is the decomposition of a metric space $\mathbb{R}^{n}$ in regions associated with a specified discrete set of points.

Let $S=\left\{s_{1}, s_{2}, \ldots, s_{\nu}\right\}$ be a collection of $\nu$ points in $\mathbf{R}^{n}$. For each point $s_{i}$ a set $V_{i}$ is associated such that $\bigcup_{i} V_{i}=\mathbf{R}^{n}$. The definition of $V_{i}$ will be:

$$
V_{i}=\left\{x \in \mathbf{R}^{n} \mid\left\|x-v_{i}\right\|_{2} \leq\left\|x-v_{i}\right\|_{2}, \forall j \neq i\right\}
$$

It can be observed that each frontier of $V_{i}$ is part of the bisection hyperplane between $s_{i}$ and one of the neighbor points $s_{j}$. As a consequence of this fact, the regions $V_{i}$ are polyhedrons. Globally, the Voronoi partition is a decomposition of space $\mathbf{R}^{n}$ in $\nu$ polyhedral regions.

\section{Nonparameterized case}

Notation:

$\mathfrak{F}(X) \quad$ The frontier of a compact set $X$

$\operatorname{Int}(X) \quad$ The interior of a compact set $X$

Consider nonparameterized optimization problem with mixed type of constraints:

$$
\begin{gathered}
\mathbf{k}_{u}^{*}=\underset{\mathbf{k}_{u}}{\arg \min } 0.5 \mathbf{k}_{u}^{T} \mathbf{k}_{u}+c^{T} \mathbf{k}_{u} \\
\left\{\begin{array}{c}
h\left(\mathbf{k}_{u}\right) \leqslant 0 \\
A_{i n} \mathbf{k}_{u} \leq b_{i n}+B_{i n} x
\end{array}\right.
\end{gathered}
$$

In relation with the feasible domain $D$ of this optimization problem one can define:

$\mathfrak{R}_{L}(D) \quad$ The set of linear constraints in the definition of $D$

$\mathfrak{R}_{N L}(D)$ The set of nonlinear constraints in the definition of $D$

$\mathfrak{S}\left(\mathfrak{R}_{*}, \mathbf{k}_{u}\right)$ The subset of constraints in $\mathfrak{R}_{*}$ (either $\mathfrak{R}_{L}$ either $\mathfrak{R}_{N L}$ ) saturated by the vector $\mathbf{k}_{u}$

$\mathfrak{B}\left(\mathfrak{R}_{*}, \mathbf{k}_{u}\right)$ The subset of constraints in $\mathfrak{R}_{*}$. violated by the vector $\mathbf{k}_{u}$

Algorithm:

1) Obtain a set of points $(\mathcal{V})$ on the frontier of the feasible domain $D$

2) Construct the convex hull $\mathcal{C}_{\mathcal{V}}$

3) Split the set $\mathcal{V}$ as $\widetilde{\mathcal{V}} \cup \overline{\mathcal{V}}_{L} \cup \overline{\mathcal{V}}_{N L} \cup \widehat{\mathcal{V}}$

- $\tilde{\mathcal{V}} \in \mathfrak{F}\left(\mathcal{C}_{\mathcal{V}}\right)$ and $\mathcal{C}_{\mathcal{V}}=\mathcal{C}_{\mathcal{V} \backslash \tilde{\mathcal{V}}}$ (those points in $\mathcal{V}$ which lie on the frontier of $\mathcal{C}_{\mathcal{V}}$ but are not vertices); 
- $\mathcal{V}_{L} \in \mathcal{V} \backslash \widetilde{\mathcal{V}}, \mathcal{V}_{L} \in \mathfrak{F}\left(\mathcal{C}_{\mathcal{V}}\right)$ and $\mathcal{V}_{L}$ saturate at least one linear constraint in (31)

- $\mathcal{V}_{N L} \in \mathcal{V} \backslash \widetilde{\mathcal{V}}, \mathcal{V}_{N L} \in \mathfrak{F}\left(\mathcal{C}_{\mathcal{V}}\right)$ and $\mathcal{V}_{N L}$ saturate only nonlinear constraints in (31)

- $\widehat{\mathcal{V}} \in \mathfrak{I n t}\left(\mathcal{C}_{\mathcal{V}}\right)$

4) Construct the dual representation of $\mathcal{C}_{\mathcal{V}}$. This will be represented as an intersection of halfspaces $\mathcal{H}$.

5) Split $\mathcal{H}$ in $\overline{\mathcal{H}} \cup \widehat{\mathcal{H}}$

- $\widehat{\mathcal{H}} \subset \mathcal{H}$ such that $\exists x \in \mathcal{C}_{\mathcal{V}}$ with $\mathfrak{S}(\widehat{\mathcal{H}}, x) \neq \emptyset$ and $\mathfrak{B}\left(\Re_{N L}, x\right) \neq \emptyset$

- $\overline{\mathcal{H}}=\mathcal{H} \backslash \widehat{\mathcal{H}}$

6) Project the unconstrained optimum $\mathbf{k}_{u}=-c$ on $\mathcal{C}_{\mathcal{V}}$ :

$$
\mathbf{k}_{u}^{*} \leftarrow \operatorname{Proj}_{\mathcal{C}_{\mathcal{V}}}\{-c\}
$$

(as it was illustrated in figure 1c)

7) If $\mathbf{k}_{u}^{*}$ saturates a subset of constraints $\mathcal{K} \subset \widehat{\mathcal{H}}$

a) Retain the set of points:

$$
\begin{array}{r}
S=\left\{v \in \widehat{\mathcal{V}} \mid \forall \mathbf{k}_{u} \in \mathcal{C}_{\mathcal{V}} \text { s.t. } \mathfrak{S a t}\left(\widehat{\mathcal{H}}, \mathbf{k}_{u}\right)=\mathcal{K} ;\right. \\
\left.\mathfrak{B}\left(\mathfrak{R}_{N L}, \mathbf{k}_{u}\right)=\mathfrak{S a t}\left(\mathfrak{R}_{N L}, v\right)\right\}
\end{array}
$$

b) Construct the Voronoi partition for the collection of points in $S$

c) Position $\mathbf{k}_{u}^{*}$ w.r.t. this partition and map the suboptimal solution $\mathbf{k}_{u}^{*} \leftarrow v$ where $v$ is the vertex corresponding to the active region

8) If the quality of the solution is not satisfactory, improve the distribution of the points $\mathcal{V}$ by augmenting the resolution around $\mathbf{k}_{u}^{*}$ and restart from (2).

\section{E. Explicit solution - taking into account the parametriza- tion}

Consider now the multiparametric optimization:

$$
\begin{gathered}
\mathbf{k}_{u}^{*}=\underset{\mathbf{k}_{u}}{\arg \min } 0.5 \mathbf{k}_{u}^{T} H \mathbf{k}_{u}+\mathbf{k}_{u}^{T} F x \\
\left\{\begin{array}{c}
h\left(x, \mathbf{k}_{u}\right) \leqslant 0 \\
A_{i n} \mathbf{k}_{u} \leq b_{\text {in }}+B_{\text {in }} x
\end{array}\right.
\end{gathered}
$$

and the set:

$$
D=\left\{\left[\begin{array}{c}
\mathbf{k}_{u} \\
x
\end{array}\right] \in \mathbb{R}^{m N_{y}+n} \mid \begin{array}{c}
h\left(x, \mathbf{k}_{u}\right) \leqslant 0 \\
A_{\text {in }} \mathbf{k}_{u} \leq b_{\text {in }}+B_{\text {in }} x
\end{array}\right\}
$$

Algorithm:

1) Grid the parameters space $\mathbb{R}^{n}$ and retain the feasible nodes $\mathcal{G}$

2) Obtain in the extended argument+parameters space a set of points $\left(\mathcal{V}_{\mathcal{G}}\right)$ lying on the frontier of $D$

3) Construct the convex hull $\mathcal{C}_{\mathcal{V}}$ for the points in $\mathcal{V}_{\mathcal{G}}$

4) Split the set $\mathcal{V}_{\mathcal{G}}$ as $\widetilde{\mathcal{V}} \cup \overline{\mathcal{V}}_{L} \cup \overline{\mathcal{V}}_{N L} \cup \hat{\mathcal{V}}$

- $\tilde{\mathcal{V}} \in \mathfrak{F}\left(\mathcal{C}_{\mathcal{V}}\right)$ and $\mathcal{C}_{\mathcal{V}}=\mathcal{C}_{\mathcal{V} \backslash \tilde{\mathcal{V}}}$ (those points in $\mathcal{V}$ which lie on the frontier of $\mathcal{C}_{\mathcal{V}}$ but are not vertices);

- $\mathcal{V}_{L} \in \mathcal{V}_{\mathcal{G}} \backslash \widetilde{\mathcal{V}}, \mathcal{V}_{L} \in \mathfrak{F}\left(\mathcal{C}_{\mathcal{V}}\right)$ and $\mathcal{V}_{L}$ saturate at least one linear constraint in (31)

- $\mathcal{V}_{N L} \in \mathcal{V}_{\mathcal{G}} \backslash \widetilde{\mathcal{V}}, \mathcal{V}_{N L} \in \mathfrak{F}\left(\mathcal{C}_{\mathcal{V}}\right)$ and $\mathcal{V}_{N L}$ saturate only nonlinear constraints in (31)

- $\widehat{\mathcal{V}} \in \mathfrak{I n t}\left(\mathcal{C}_{\mathcal{V}}\right)$
5) Construct the dual representation of $\mathcal{C}_{\mathcal{V}}$. This will be represented as a intersection of halfspaces $\mathcal{H}$.

6) Split $\mathcal{H}$ in $\overline{\mathcal{H}} \cup \widehat{\mathcal{H}}$

- $\widehat{\mathcal{H}} \subset \mathcal{H}$ such that $\exists x \in \mathcal{C}_{\mathcal{V}}$ with $\mathfrak{S}(\widehat{\mathcal{H}}, x) \neq \emptyset$ and $\mathfrak{B}\left(\mathfrak{R}_{N L}, x\right) \neq \emptyset$

- $\overline{\mathcal{H}}=\mathcal{H} \backslash \widehat{\mathcal{H}}$

7) Project the set

$U=\left\{\left[\begin{array}{c}\mathbf{k}_{u} \\ x\end{array}\right] \mid\left[\begin{array}{c}\mathbf{k}_{u} \\ x\end{array}\right]=\left[\begin{array}{c}H^{-1} F \\ I\end{array}\right] x, \forall x \in \mathbb{R}^{n}\right\}$

on $\mathcal{C}_{\mathcal{V}}$ :

$$
U^{*} \leftarrow \operatorname{Proj}_{\mathcal{C}_{\mathcal{V}}} U
$$

8) If $\exists x_{0}$ such that the point:

$$
\left[\begin{array}{c}
\mathbf{k}_{u}^{*} \\
x_{0}
\end{array}\right]=U^{*} \cap\left\{\left[\begin{array}{c}
\mathbf{k}_{u} \\
x
\end{array}\right] \mid x=x_{0}\right\}
$$

saturates a subset of constraints

$$
\mathcal{K}\left(x_{0}\right)=\mathfrak{S}\left(\mathcal{H},\left[\begin{array}{l}
\mathbf{k}_{u} \\
x_{0}
\end{array}\right]\right) \subset \widehat{\mathcal{H}}
$$

then:

a) Construct

$$
\begin{gathered}
U_{N L}\left(x_{0}\right)=\left\{\left[\begin{array}{c}
\mathbf{k}_{u} \\
x
\end{array}\right] \in U \mid\left[\begin{array}{c}
\mathbf{k}_{u}^{*} \\
x
\end{array}\right] \in U^{*}\right. \\
\text { t.q. } \left.\mathfrak{S}\left(\mathcal{H},\left[\begin{array}{c}
\mathbf{k}_{u} \\
x_{0}
\end{array}\right]\right)=\mathcal{K}\left(x_{0}\right)\right\}
\end{gathered}
$$

b) Perform:

$$
U^{*}=U^{*} \backslash\left\{\left[\begin{array}{c}
\mathbf{k}_{u} \\
x
\end{array}\right] \mid \mathfrak{S}\left(\mathcal{H},\left[\begin{array}{c}
\mathbf{k}_{u} \\
x_{0}
\end{array}\right]\right)=\mathcal{K}\left(x_{0}\right)\right\}
$$

c) Retain the set of points:

$$
\begin{array}{r}
S=\left\{v \in \widehat{\mathcal{V}} \mid \forall\left[\begin{array}{c}
\mathbf{k}_{u} \\
x
\end{array}\right] \in \mathcal{C}_{\mathcal{V}} \quad\right. \text { with } \\
\mathfrak{S}\left(\widehat{\mathcal{H}},\left[\begin{array}{c}
\mathbf{k}_{u} \\
x
\end{array}\right]\right)=\mathcal{K}\left(x_{0}\right) \Rightarrow \\
\left.\mathfrak{B}\left(\Re_{N L}, x\right)=\mathfrak{S}\left(\Re_{N L}, v\right)\right\}
\end{array}
$$

d) Construct the Voronoi partition for the collection of points in $S$

e) Position $U_{N L}\left(x_{0}\right)$ w.r.t. this partition and map the suboptimal solution $U_{N L}^{*}\left(x_{0}\right) \leftarrow U_{N L}\left(x_{0}\right)$ by using the vertex $v$ for each active region.

$$
\left[\begin{array}{c}
\mathbf{k}_{u}^{*} \\
x
\end{array}\right]=v \leftarrow\left[\begin{array}{c}
\mathbf{k}_{u} \\
x
\end{array}\right]
$$

else: jump to (10)

9) Return to point (8)

10) If the quality of the solution is not satisfactory, improve the distribution of the points $\mathcal{V}_{\mathcal{G}}$ and restart from (2). 


\section{NUMERICAL EXAMPLE}

A. Simple convex multiparametric nonlinear program

Consider the discrete-time linear system:

$$
x_{t+1}=\left[\begin{array}{cc}
0.9 & 1 \\
0 & 1
\end{array}\right] x_{t}+\left[\begin{array}{c}
1 \\
-1
\end{array}\right] u_{t}
$$

and a predictive control law with a prediction horizon of three sampling times and a control horizon of two steps. A nonlinear set of constraints will be also considered:

$$
\left\{\begin{array}{l}
\sum_{k=0}^{2} u_{t+k}^{2} \leq 1 \\
\sum_{k=0}^{2} u_{t+k}^{2} \leq \ln \left(\left[\begin{array}{cc}
0 & 1
\end{array}\right] x_{t}+1\right) \\
{\left[\begin{array}{cc}
0 & 1
\end{array}\right] x_{t+k} \geq 0 ; k=0,1,2}
\end{array}\right.
$$

It is obvious that the topology of the feasible domain is changing with the system dynamics, which means that the state vector represents in fact a parameter. More precisely, in our case only the second component of the state, $x_{t}$ is influencing the shape of the feasible domain and thus one can draw this dependence on the parameter as in figure 3. Further this parameterized convex shape can be approxi-

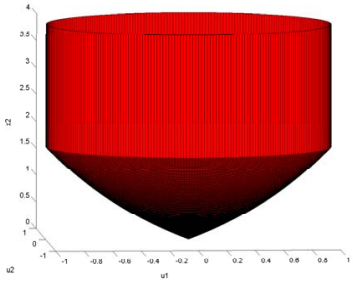

Fig. 3. The nonlinear dependence of the feasible domain on the parameters

mated with a set of parameterized linear inequalities and obtain a double description of a parameterized polyhedron as in figure 4(a). A precutting in zones with regular shape (figure 4(b)) can help in the development of explicit solution due to the important degree of redundancy.

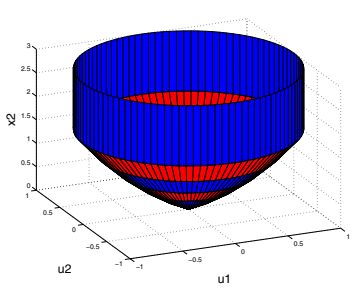

(a)

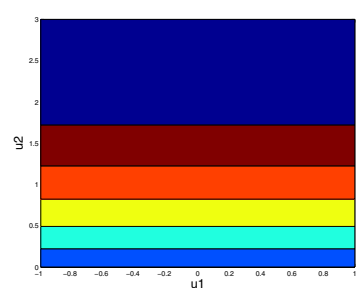

(b)
Fig. 4. (a) The approximation by a parameterized polyhedron; (b) Regions in the parameters' space corresponding to redundancy-free constraints sets.

Finally the nonlinear MPC law for the system (33) and the constraints (34) can be approximated by the explicit solution found in terms of a piecewise linear control law as in figure 5.

\section{B. Example of non-Convex problem}

Consider the MPC problem implemented using the first control action of the optimal sequence:

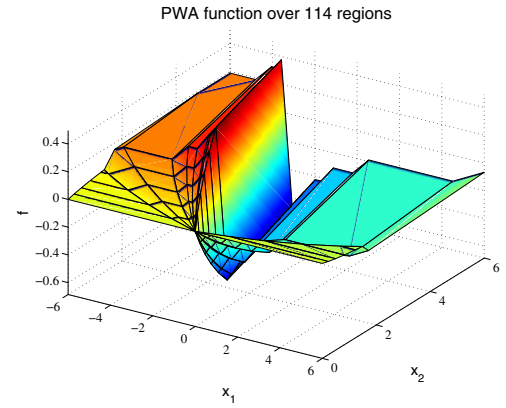

Fig. 5. Explicit solution as a piecewise linear function.

$$
\begin{gathered}
k_{u}^{*}=\underset{k_{u}}{\arg \min } \sum_{i=0}^{N_{y}-1} x_{t+k \mid t}^{T} Q x_{t+k \mid t}+u_{t+k \mid t}^{T} R u_{t+k \mid t}+ \\
\quad+x_{t+N_{y} \mid t}^{T} P x_{t+N_{y} \mid t}
\end{gathered}
$$

with

$Q=\left[\begin{array}{cc}10 & 0 \\ 0 & 1\end{array}\right] ; R=\left[\begin{array}{ll}2 & 0 \\ 0 & 3\end{array}\right] ; P=\left[\begin{array}{cc}13.73 & 2.46 \\ 2.46 & 2.99\end{array}\right]$

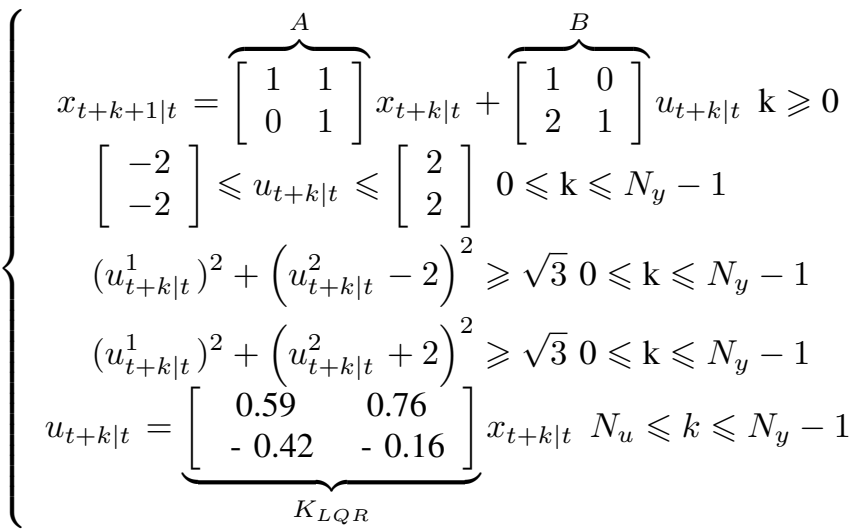

One can observe the presence of both linear and nonlinear constraints. By following the previous algorithm, in the first stage, the partition of the state space is performed by considering only the linear constraints (figure 6(a)).

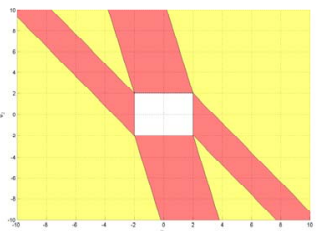

(a)

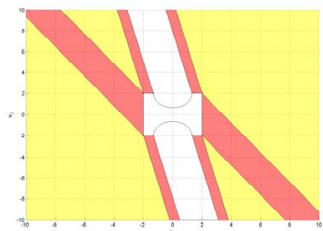

(b)
Fig. 6. a) Partition of the arguments space (linear constraints only). b) Retention of the regions with feasible linear projections.

Each such region correspond with a specific projection law. By simply verifying the regions where this projection law obey the nonlinear constraints, the exact part of the explicit solution is obtained (figure 6(b)).

Further, a distribution of points on the nonlinear frontier of the feasible domain has to be obtained and the 
associated Voronoi partition obtained. By superposing it to the regions non covered at the previous step one obtain a complete covering of the arguments space. Figure 7(a) depicts such a complete partition for distribution of 10 points for each nonlinear constraint. Figure 7(b) augments the density to 100 points.

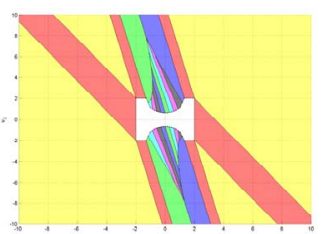

(a)

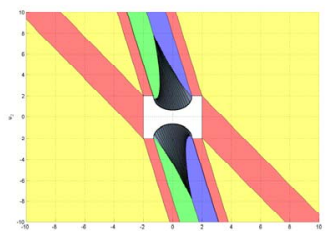

(b)
Fig. 7. a) Partition of the arguments space (nonlinear case) - 10 point per nonlinear constraint. b) Partition of the arguments space (nonlinear case) - 100 points per nonlinear constraint.

By correspondence, the figures 8(a) and 8(b) describe the partition of the state space for the explicit solution.

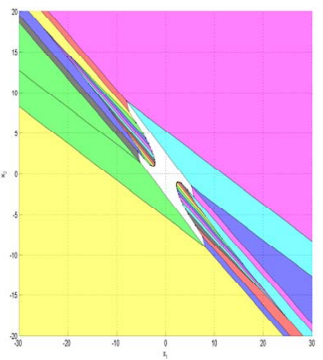

(a)

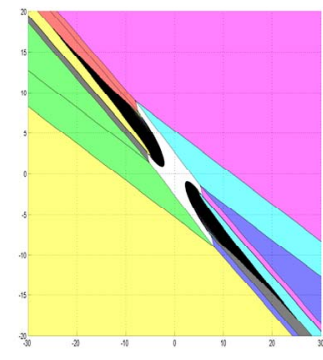

(b)
Fig. 8. a) Partition of the state space - 10 points per nonlinear constraint. b) Partition of the state space - 100 points per nonlinear constraint.

Finally the complete explicit solution for the two cases are described in figures 9(a) and 19(b). The discontinuities are observable as well as the increase in resolution over the nonlineairity with the augmentation of the number of points in the Voronoi partition.

In order to give a give an image of the complexity it must be said that the explicit solutions have 31 and 211 regions respectively and the computational effort was less than $2 s$ in the first case and $80 s$ in the second case, mainly spent in the construction of supplementary regions in the Voronoi partition.

\section{CONCLUSIONS}

The construction of explicit solution for receding horizon optimal control problems was presented with a special attention to the nonlinear constraints handling mechanism. The geometrical approach exploits the topology of the feasible domain. As long as the unconstraint optimum is projected on a linear set of constraints one obtains the exact solutions. For the regions where the unconstraint optimum violates nonlinear constraints, a technique based on Voronoi partition allows the construction of approximate explicit solution.

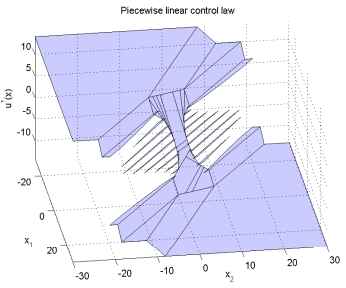

(a)

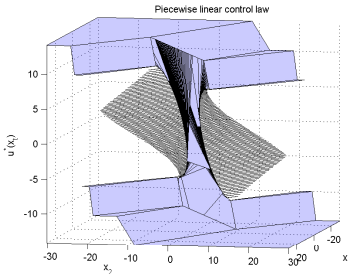

(b)
Fig. 9. a) Explicit control law - 10 points per nonlinear constraint. b) Explicit control law - 100 points per nonlinear constraint.

The degree of suboptimality for the solution under active nonlinear constraints is directly related to the distribution of the extreme points on the frontier of the feasible domain. The presented algorithm can be used in a recursive manner in order to improve the quality of the explicit solution. However this is done with the price of an increased complexity for the piecewise control law.

\section{REFERENCES}

[1] G.C. Goodwin, M.M. Seron and J.A. De Dona, " Constrained Control and Estimation". Springer-Verlag, London, 2004.

[2] E.F. Camacho and C. Bordons, "Model Predictive Control", Springer, 1999.

[3] D.Q. Mayne, J.B. Rawlings, C.V. Rao, P.O.M. Scockaert, Constrained model predictive control: Stability and optimality, Automatica, Vol.36, pp. 789-814, 2000.

[4] T.Gal and J.Nedoma, Multiparametric Linear Programming. Management Science, vol. 18, pp. 406-442, 1972.

[5] P. Tondel, T. Johansen and A. Bemporad, "An algorithm for multiparametric quadratic programming and explicit MPC solutions", Proc. 40th IEEE CDC, 2001.

[6] I.A. Fotiou , P. Rostalski, B. Sturmfelds and M. Morari, "An algebraic geometry approach to nonlinear parametric optimization in control", American Control Conference, Minneapolis, 2005.

[7] A. Grancharova, P. Tøndel and T. A. Johansen, "Computational Aspects of Approximate Explicit Nonlinear Model Predictive Control", NMPC'05 - International Workshop on Assessment and Future Directions of Nonlinear Model Predictive Control, Freudenstadt-Lauterbad, Germany, 2005.

[8] S. Olaru, D. Dumur, "Explicit constrained model predictive control. The influence of constraints redundancy", 5th IEEE International Conference on Control and Automation, Budapest, Hongrie, 26-29 June 2005

[9] Motzkin, T.S., Raiffa, H., Thompson, G.L. and Thrall R.M., "The Double Description Method", (1953) republished in Theodore S. Motzkin: Selected Papers, Birkhauser, Boston, 1983.

[10] Leverge, H., "A note on Chernikova's algorithm", Technical Report, IRISA 635, 1994.

[11] Loechner, V. and Wilde, D. K., "Parameterized polyhedra and their vertices". Int. J. of Parallel Programming, 25(6), 1997.

[12] A. Schrijver, "Theory of linear and integer programming", John Wiley and Sons, NY, 1986.

[13] Wilde, D.K., "A library for doing polyhedral operations", Technical report, IRISA 785, 1993.

[14] Bemporad, A., Morari, M., Dua, V., Pistikopoulos, E.; "The explicit linear quadratic regulator for constrained systems", $\mathrm{Au}$ tomatica, vol.38, pp. 3-20, (2002).

[15] Seron, M.M., Goodwin, G.C. and De Dona J.A., "Characterisation of Receding Horizon Control for Constrained Linear Systems", Asian Journal of Control, vol. 5-2, pp. 271-286, 2003.

[16] Olaru, S. and Dumur D., "A Parameterized Polyhedra Approach for Explicit Constrained Predictive Control", 43rd IEEE Conference on Decision and Control , 2004. 